\title{
O Perfil da saúde das mulheres e os motivos que as levam a evitar a gestação
}

\author{
Women's health and the reasons that lead them to avoid pregnancy \\ Salud de la mujer y las razones que las llevan a evitar el embarazo
}

Glauciane Marques de Assis Berteloni ${ }^{1 *}$, Maria Elisa Wotsazek Cestari², Thelma Malagutti Sodré2.

\section{RESUMO}

Objetivo: Identificar o perfil da saúde das mulheres usuárias da internet e os motivos que as levaram a evitar a gestação. Métodos: Trata-se de uma pesquisa quantitativa, transversal e descritiva, com coleta de dados por formulário divulgado em rede social on-line. Resultados: Participaram 1071 mulheres, entre 14 e 29 anos, mais de $40 \%$ possuíam ensino superior completo, $87,6 \%$ eram nuligestas e 40,8\% tinham histórico gestacional (MCHO). Os motivos que as levaram a evitar a gestação foram: possuir a quantidade de filhos desejada $(34,1 \%)$; condições financeiras desfavoráveis $(16,2 \%)$; desejo de engravidar posteriormente $(39,1 \%)$; planos de concluir os estudos $(25,3 \%)$ e não-maternidade $(20,6 \%)$. Os métodos anticoncepcionais mais utilizados foram o hormonal oral $(43,6 \%)$ e de barreira $(34,5 \%)$. Conclusão: O perfil das mulheres da internet colabora para maiores possibilidades de empoderamento e autonomia devido ao acesso à informação. As mulheres continuam em adaptação aos novos modelos de vida, apresentam motivos relevantes para evitar a gestação e utilizam métodos reversíveis para postergar a maternidade. As mulheres devem ter condições de escolha informada em relação ao seu planejamento reprodutivo, para isso, os serviços de saúde devem garantir acesso aos métodos e informações adequadas sobre a contracepção, para prevenção de agravos à saúde da mulher.

Palavras-chave: Anticoncepção, Saúde da mulher, Planejamento familiar, Gravidez, Internet.

\begin{abstract}
Objective: This paper aims to identify the profile of women who are internet users and the reasons that led them to avoid pregnancy. Methods: This is a quantitative, cross-sectional and descriptive research with data collection by form published on online social network. Results: There were 1071 women participating, between 14 and 29 years of age, more than $40 \%$ had completed higher education, $87.6 \%$ were nulliparous and $40.8 \%$ had a gestational history (women with obstetric history - WOH). The reasons that led them to avoid pregnancy were having the desired number of children (34.1\%); unfavorable financial conditions (16.2\%); desire of getting pregnant later (39.1\%); plans to finish studies (25.3\%) and non-maternity $(20.6 \%)$. The most commonly used contraceptive methods were oral - hormonal method (43.6\%) and barrier method (34.5\%). Conclusion: The profile of women who are Internet users contributes to greater possibilities of empowerment and autonomy due to their access to information. Women continue to adapt to new life patterns, give relevant reasons to avoid pregnancy and use reversible methods in order to delay motherhood. Women must have conditions of informed choice in relation to their reproductive planning; to this end, health services must ensure access to adequate methods and information on contraception for the prevention of worsening women's health.
\end{abstract}

Keywords: Anticonception, Women's health, Family planning, Pregnancy, Internet.

\footnotetext{
1 Unidade Materno Infantil do Complexo Hospital de Clínicas da Universidade Federal do Paraná (UMI-CHC-UFPR), Curitiba-PR. *E-mail: glauciane_berteloni@hotmail.com

2 Departamento de Enfermagem da Universidade Estadual de Londrina (UEL), Londrina-PR.
} 


\section{RESUMEN}

Objetivo: Identificar el perfil de las mujeres usuarias de internet y las razones que las llevan a evitar el embarazo. Métodos: Se trata de una investigación cuantitativa, transversal y descriptiva con recolección de datos por formulario publicado en la red social en línea. Resultados: Participaron 1071 mujeres, entre 14 y 29 años de edad, más del $40 \%$ con estudio superior completo, $87,6 \%$ eran nulíparas y el $40,8 \%$ tenía histórico gestacional (MCHO). Los motivos que las llevaron a evitar el embarazo fueron: poseer la cantidad de hijos deseada $(34,1 \%)$; condiciones financieras desfavorables $(16,2 \%)$; deseo de embarazar posteriormente $(39,1 \%)$; planes de concluir los estudios $(25,3 \%)$ y no-maternidad $(20,6 \%)$. Los métodos anticoncepcionales más utilizados fueron el hormonal oral $(43,6 \%)$ y de barrera $(34,5 \%)$. Conclusión: El perfil de las mujeres en Internet contribuye a mayores posibilidades de empoderamiento y autonomía debido al acceso a la información. Las mujeres continúan en proceso de adaptación a los nuevos modelos de vida, presentan motivos relevantes para evitar la gestación y utilizan métodos reversibles para postergar la maternidad. Las mujeres deben tener condiciones de elección informada con relación a su planeamiento reproductivo, para eso, los servicios de salud deben garantizar el acceso a los métodos e informaciones adecuadas sobre la contracepción, para la prevención de daños a la salud de la mujer.

Palabras clave: Anticoncepción, Salud de la mujer, Planificación familiar, Embarazo, Internet.

\section{INTRODUÇÃO}

A saúde da mulher na sua integralidade evoluiu com as tecnologias em saúde disponíveis, principalmente com advento dos contraceptivos. Boa parte das mulheres passaram por um perfil tradicional de cuidados com o lar, marido e filhos à mulher com protagonismo na busca da sua independência, que investe no desenvolvimento acadêmico e profissional, com melhores condições de competir no mercado de trabalho (JUSTINO GBS, et al., 2019; CUNHA MS e VASCONCELOS MR, 2016).

As transformações sociais do papel feminino em virtude da fecundidade brasileira resultaram em uma decrescente diminuição no número de filhos, com a queda de 2,4 para 1,7 filhos por mulher no período entre 2000 a 2015 (BRASIL, 2018). A redução do número de filhos está associada ao desenvolvimento das mulheres, a modificação cultural e comportamental, desencadeando, muitas vezes, o sentimento de ambivalência do papel feminino em relação às expectativas familiares e individuais. Nesse contexto, há mulheres que optam a permanecer com seus papeis sociais de esposa, mãe, responsável pelos cuidados da família e lar, e que procuram o equilíbrio com seus estudos e sua jornada de trabalho (COSTA FA, 2018; MACHADO JSA E PENNA CMM, 2016).

Para diminuir o número de filhos as mulheres recorreram ao uso de métodos anticoncepcionais. Dos métodos disponíveis a partir da década de 50 no mundo, os mais utilizados são o contraceptivo hormonal oral, esterilização cirúrgica feminina e o preservativo masculino.

O uso de anticoncepcionais faz parte do planejamento reprodutivo (PR), anteriormente conhecido como planejamento familiar, que foi definido como uma das políticas de ações da saúde reprodutiva para garantir os direitos de limitação ou aumento de filhos pelo casal ou indivíduo, com o espaçamento entre as gestações, bem como facilitar o acesso à informação e orientação sobre a concepção (RODRIGUES LSA, et al., 2014; SILVA MMJ, et al., 2017).

No Brasil, as políticas públicas sobre a saúde da mulher iniciaram-se em 1984, antes da criação do Sistema Único de Saúde (SUS), com o Programa de Atenção Integral à Saúde da Mulher (PAISM) que ampliava os cuidados à mulher para além do foco reprodutivo. Dentre as ações do PAISM, foram elaborados um conjunto de medidas de Planejamento Familiar no âmbito da saúde pública. Mas somente a partir da Constituição Federal (1988), foi legalmente descrito o Programa Planejamento Familiar, que recebeu critérios da sua realização na Lei no 9.263, de 1996, que estabeleceu formas para sua execução com caráter principalmente educacional e informativo, sem coerção de controle populacional (BRASIL, 1988; BRASIL, 1996; BRASIL; 2013). 
Entretanto, mais de $50 \%$ das gestações são declaradas, ainda, como não planejadas, ou não previstas, podendo ser resultado da falha ou ausência do acesso à informação, uso incorreto ou inexistente de contraceptivos, como também de fatores socioculturais, religiosos ou até mesmo do desejo inconsciente de gestar (MARCOS CM, 2017; BASTOS LL, et al,. 2014; BRANDÃO ER e CABRAL CS, 2017).

No cenário das gestações não planejadas há também a necessidade de intervenção quanto à morbidade e mortalidade materna evitável, e também em relação ao abortamento, por não ser um direito reprodutivo legalizado no Brasil, é legal apenas em caso de vítimas de violência sexual ou abortamentos previstos como no caso da anencefalia (AZEVEDO AF, 2017; OLIVEIRA ECS, 2018).

Neste contexto, a mulher que não deseja a gestação oriunda de uma relação sexual consentida torna-se vulnerável às complicações pós-abortamento clandestino e inseguro, como por exemplo, as complicações hemorrágicas e as infecções puerperais. Diante disto, e em associação ao estilo de vida moderno, a anticoncepção vem como atuação dos serviços de saúde para a prevenção de agravos à saúde da mulher e possibilidade de escolha em relação a sua própria saúde reprodutiva (DINIZ D, et al., 2016).

A mulher pode utilizar os métodos anticoncepcionais reversíveis durante boa parte da vida, o que permite a postergação da maternidade até o momento ideal para uma gestação, se desejar, podendo haver o planejamento reprodutivo atrelado com a estabilidade financeira e emocional, a conclusão dos estudos e a realização profissional (OLSEN JM, et al., 2018).

O estilo de vida moderno pode trazer também nova forma de realizar o PR, no que diz respeito ao uso da internet pela busca da informação em saúde. A mudança do perfil das mulheres e o desenvolvimento tecnológico corroboram na estratégia do uso da internet, como ferramenta de acesso livre, cada vez mais utilizada na área da saúde pelas mulheres (MORETTI FA, et al., 2012).

A internet é uma poderosa aliada para trocar aprendizado e vivências, proporcionando possível melhora na qualidade de vida e contribuindo com a interação social, estimulação da autonomia, empoderamento das mulheres e proteção dos direitos reprodutivos.

O conteúdo disponível pode não ser claro à interpretação ou de qualidade, e a mulher deve ter senso de crítica com condições suficientes de entendimento, e manter o acesso aos profissionais de saúde (PEREIRA NA, et al., 2016; SEABRA ACCM e DUSSE F, 2017). O objetivo deste estudo foi identificar o perfil da saúde das mulheres usuárias da internet e os motivos que as levaram a evitar a gestação.

\section{MÉTODOS}

O delineamento deste estudo teve como base um desenho quantitativo, transversal e exploratório. A população foi de mulheres brasileiras usuárias da internet, com amostra probabilística e acidental de 1071 mulheres. O formulário eletrônico foi divulgado por meio de uma conta/perfil que participou de aproximadamente 500 grupos do Facebook com temáticas variadas, com abrangência das comunidades virtuais de todos estados brasileiros. Foi adotado como critério de exclusão as mulheres brasileiras que declararam residência no exterior.

O formulário eletrônico foi acompanhado por uma mensagem de convite às mulheres para participarem da pesquisa de forma voluntária, sem remuneração ou ônus. A composição do formulário disponibilizado foi predominantemente por perguntas fechadas, com variáveis socioeconômicas, demográficas, hábitos de vida, presença de morbidade, histórico obstétrico, uso de métodos anticoncepcionais e os motivos para evitar a gestação.

A elaboração do formulário ocorreu por meio do aplicativo online Google Docs, sua validação realizou-se por um teste piloto nas redes sociais com 15 mulheres e após, a amostra teste foi excluída dos resultados da pesquisa.

Para participar desta pesquisa, as mulheres tiveram acesso na primeira página do formulário eletrônico ao Termo de Consentimento Livre e Esclarecido (TCLE), conforme os requisitos da Resolução Conselho Nacional de Saúde № 466/ 2012. Logo após o aceite do TCLE, de visualização facilitada no ambiente do 
aplicativo, as mulheres tinham acesso às perguntas, ou caso, não aceitassem participar, eram direcionadas à página final de agradecimento e as formas de contato com as pesquisadoras para mais informações sobre a pesquisa.

A coleta de dados aconteceu via internet, com divulgação da pesquisa pela rede social Facebook, por meio de um formulário com divulgação em todo o território nacional, de abril a novembro do ano de 2014 . O estudo aconteceu mediante o Parecer do Comitê de Ética em Pesquisa da instituição e Certificado de Apresentação para Apreciação Ética no 27110614.0.0000.5231.

Para a análise dos dados foi exportada a base de dados do aplicativo do Google Docs em uma planilha do programa Excel for Windows, tabulados e analisados no programa Statistical Package for Social Science for Windows versão 20. Foi utilizado tratamento estatístico descritivo com nível de confiança de $95 \%$ e margem de erro de $5 \%$, obtidos variáveis com proporções de frequências, desvio padrão, e tabela de referência cruzada com uso de proporções, porcentagens e Teste qui-quadrado de Person ( $p$ ). Os resultados foram elaborados em análise cruzada entre nuligestas e mulheres com histórico obstétrico (MCHO). Definiuse como nuligesta mulheres com ausência de histórico gestacional declarado (SCAVUZZI A, et al., 2016).

\section{RESULTADOS}

Participaram deste estudo 1071 mulheres usuárias da internet com idade mínima de 14 e máxima de 49 anos, com moda de 24 anos, média de 26 anos, e desvio padrão de 6,42 anos. O estudo foi predominante entre mulheres jovens em $87 \%$, entre 14 a 29 anos, nuligestas, $41 \%$ das $\mathrm{MCHO}$ e $65 \%$ das nuligestas com ensino superior, declararam possuir companheiro em $83,4 \%$ das $\mathrm{MCOH}$ e $58,6 \%$ das nuligestas. Esta pesquisa não teve a intenção de quantificar número de filhos por mulheres e a idade em que ocorreram as gestações (Tabela 1).

Tabela 1 - Caracterização social das mulheres, $\mathrm{n}=1071$. Brasil, 2014.

\begin{tabular}{|c|c|c|c|c|c|}
\hline Variáveis & $\begin{array}{c}\mathrm{MCHO} \\
\mathbf{N}\end{array}$ & $\%$ & $\begin{array}{c}\text { Nuligestas } \\
\mathbf{N}\end{array}$ & $\%$ & Valor de $\mathbf{p}^{\star}$ \\
\hline \multicolumn{6}{|l|}{ Faixa etária } \\
\hline 14-29 anos & 142 & 48,0 & 679 & 87,6 & 188,09 \\
\hline $30-49$ anos & 154 & 52,0 & 96 & 12,4 & \\
\hline \multicolumn{6}{|l|}{ Escolaridade } \\
\hline Ensino fundamental e médio & 78 & 26,4 & 57 & 7,4 & \\
\hline Ensino superior: graduação & 124 & 41,9 & 504 & 65,0 & 82,16 \\
\hline Pós-graduação & 94 & 31,8 & 214 & 27,6 & \\
\hline \multicolumn{6}{|l|}{ Região do Brasil } \\
\hline Norte & 15 & 5,1 & 22 & 2,8 & \\
\hline Nordeste & 39 & 13,2 & 98 & 12,6 & \\
\hline Centro Oeste & 22 & 7,4 & 28 & 3,6 & 15,08 \\
\hline Sudeste & 122 & 41,2 & 297 & 38,3 & \\
\hline Sul & 98 & 33,1 & 330 & 42,6 & \\
\hline \multicolumn{6}{|l|}{ Situação conjugal } \\
\hline Sem companheiro & 49 & 16,6 & 321 & 41,4 & \\
\hline Com companheiro & 247 & 83,4 & 454 & 58,6 & 58,56 \\
\hline Total & 296 & - & 775 & - & - \\
\hline
\end{tabular}

Fonte: Berteloni GMA, et al., 2014.

Baseado em salários mínimos grande parte concentram a renda entre 1 a 3 salários em $37,5 \%$ das $\mathrm{MCHO}$ e $30,2 \%$ das nuligestas. As formações familiares em média foram de três pessoas em aproximadamente $40 \%$ das $\mathrm{MCHO}$ e $31,2 \%$ entre as nuligestas. A maioria das mulheres são usuárias do sistema público de saúde do Brasil entre $71,6 \% \mathrm{MCHO}$ e $61,7 \%$ das nuligestas. Quanto a situação ocupacional, $79,4 \%$ das $\mathrm{MCHO}$ exercem atividades remuneradas e entre nuligestas 52,5\% não exercem atividades remuneradas (Tabela 2). 
Tabela 2 - Caracterização econômica das mulheres, n= 1071. Brasil, 2014.

\begin{tabular}{cccccc}
\hline Variáveis & MCHO & N & Nuligestas & $\%$ & Valor de $\mathbf{p}^{*}$ \\
\hline Renda familiar & & & & & \\
\hline Até um salário mínimo (SM) & 11 & 3,7 & 20 & 2,6 & \\
> um - três SM & 111 & 37,5 & 234 & 30,2 & \\
> três - cinco SM & 69 & 23,3 & 183 & 23,6 & 13,55 \\
> Cinco - dez SM & 71 & 24,0 & 182 & 23,5 & \\
$>$ Dez ou mais SM & 34 & 11,5 & 156 & 20,1 & \\
\hline
\end{tabular}

Pessoas/renda familiar

\begin{tabular}{cccccc}
\hline Uma pessoa & 11 & 3,7 & 88 & 11,4 & \\
Duas pessoas & 42 & 14,2 & 166 & 21,4 & \\
Três pessoas & 118 & 39,9 & 171 & 22,1 & 48,31 \\
Quatro pessoas & 78 & 26,3 & 242 & 31,2 & \\
Cinco ou mais pessoas & 47 & 15,9 & 108 & 13,9 & \\
\hline Sistema de saúde & & & & & 9,24 \\
\hline Sistema público & 212 & 71,6 & 478 & 61,7 & 38,3 \\
Sistema privado & 84 & 28,4 & 297 & & 87,34 \\
\hline Situação ocupacional & & & & & \\
\hline Atividade remunerada & 235 & 79,4 & 368 & 47,5 & - \\
Atividade não remunerada & 61 & 20,6 & 407 & 52,5 & - \\
\hline Total & 296 & - & 775 &
\end{tabular}

Fonte: Berteloni GMA, et al., 2014.

Quanto aos hábitos de vida, sobre o tabagismo foi referido conforme o consumo no momento da pesquisa, o mesmo critério também foi utilizado para consumo de bebidas alcoólicas e se estavam em prática de exercícios físicos.

O tabagismo estava ausente em $91,2 \%$ das $\mathrm{MCHO}$ e $93,4 \%$ das nuligestas. Sobre o consumo de bebidas alcoólicas $46,3 \%$ das $\mathrm{MCHO}$ não consumiam. Nas nuligestas o não consumo bebidas alcoólicas e consumo mensalmente foram igualmente superiores a $38 \%$ das mulheres.

O sedentarismo estava presente nos dois grupos, com $66 \%$ das $\mathrm{MCHO}$ e $54,5 \%$ das nuligestas. A alimentação fracionada estava presente em mais de $75 \%$ nos dois grupos. Em relação ao histórico de doenças, quase $80 \%$ das $\mathrm{MCHO}$ e nuligestas relataram não possuírem morbidades (Tabela 3). 
Tabela 3 - Caracterização dos hábitos de vida e morbidade das mulheres, n= 1071. Brasil, 2014.

\begin{tabular}{|c|c|c|c|c|c|}
\hline Variáveis & $\begin{array}{c}\mathrm{MCHO} \\
\mathbf{N}\end{array}$ & $\%$ & $\begin{array}{c}\text { Nuligestas } \\
\mathbf{N}\end{array}$ & $\%$ & Valor de $\mathrm{p}^{*}$ \\
\hline \multicolumn{6}{|l|}{ Tabagismo } \\
\hline Tabagista & 26 & 8,8 & 41 & 6,6 & \\
\hline Não tabagista & 270 & 91,2 & 724 & 93,4 & 1,55 \\
\hline \multicolumn{6}{|l|}{ Consumo de bebidas alcoólicas } \\
\hline Semanalmente & 48 & 16,2 & 176 & 22,7 & \\
\hline Mensalmente & 111 & 37,5 & 301 & 38,8 & 10,60 \\
\hline Não consome bebida alcoólica & 137 & 46,3 & 298 & 38,5 & \\
\hline \multicolumn{6}{|l|}{ Exercícios físicos } \\
\hline Uma a duas vezes por semana & 47 & 15,9 & 151 & 19,5 & \\
\hline Três a sete vezes por semana & 53 & 17,9 & 202 & 26,0 & 12,63 \\
\hline Sedentárias & 196 & 66,2 & 422 & 54,5 & \\
\hline \multicolumn{6}{|l|}{ Alimentação } \\
\hline Uma a três porções diárias & 73 & 24,7 & 183 & 23,6 & \\
\hline Quatro porções diárias ou mais & 223 & 75,3 & 592 & 76,4 & 0,13 \\
\hline \multicolumn{6}{|l|}{ Morbidade } \\
\hline Sim & 60 & 20,3 & 162 & 20,9 & \\
\hline Não & 236 & 79,7 & 613 & 79,1 & 0,02 \\
\hline \multicolumn{6}{|l|}{ IMC } \\
\hline Magreza $<16$ a $18,4 \mathrm{~cm}^{2}$ & 10 & 3,4 & 68 & 8,8 & \multirow{4}{*}{40,48} \\
\hline Eutrofia $>18,5$ a $24,9 \mathrm{~cm}^{2}$ & 146 & 49,3 & 492 & 63,5 & \\
\hline Sobrepeso > 25 a $29,9 \mathrm{~cm}^{2}$ & 89 & 30,1 & 136 & 17,5 & \\
\hline Obesidade $>30$ a $40 \mathrm{~cm}^{2}$ & 51 & 17,2 & 79 & 10,2 & \\
\hline Total & 296 & 100 & 775 & 100 & - \\
\hline
\end{tabular}

Fonte: Berteloni GMA, et al., 2014.

No formulário sobre o uso de métodos anticoncepcionais foi possível responder múltiplas alternativas. Outros métodos anticoncepcionais foram agrupados, devido ao quantitativo ser irrisório nesta pesquisa (implante intradérmico, diafragma e anel vaginal).

A esterilização cirúrgica corresponde tanto a laqueadura tubária como a vasectomia. Observou-se 93,9\% das $\mathrm{MCHO}$ e $86,6 \%$ das nuligestas utilizavam algum método, destes foi utilizado anticoncepcional oral em $43,6 \% \mathrm{MCHO}$ e $62,5 \%$ das nuligestas, e o preservativo entre $51,9 \%$ das nuligestas e $34,5 \%$ das $\mathrm{MCHO}$ (Tabela 4). 
Tabela 4 - Caracterização do uso de métodos anticoncepcionais entre as mulheres, n=1071. Brasil, 2014.

\begin{tabular}{|c|c|c|c|c|c|}
\hline Variáveis & $\begin{array}{c}\mathrm{MCHO} \\
\mathrm{N}\end{array}$ & $\%$ & $\begin{array}{c}\text { Nuligestas } \\
\mathbf{N}\end{array}$ & $\%$ & Valor de $\mathrm{p}^{*}$ \\
\hline \multicolumn{6}{|c|}{ Utilização método } \\
\hline Sim & 278 & 93,9 & 671 & 86,6 & \multirow[t]{2}{*}{11,42} \\
\hline Não & 18 & 6,1 & 104 & 13,4 & \\
\hline \multicolumn{6}{|l|}{ Preservativo } \\
\hline Sim & 102 & 34,5 & 402 & 51,9 & \multirow{2}{*}{25,37} \\
\hline Não & 194 & 65,5 & 373 & 48,1 & \\
\hline \multicolumn{6}{|c|}{ Anticoncepcional oral } \\
\hline Sim & 129 & 43,6 & 484 & 62,5 & \multirow{2}{*}{31,16} \\
\hline Não & 167 & 56,4 & 291 & 37,5 & \\
\hline \multicolumn{6}{|c|}{ Comportamental } \\
\hline Sim & 32 & 10,8 & 30 & 3,9 & \multirow{2}{*}{17,66} \\
\hline Não & 264 & 89,2 & 745 & 96,1 & \\
\hline \multicolumn{6}{|c|}{ Coito interrompido } \\
\hline Sim & 41 & 13,9 & 85 & 11,0 & \multirow{2}{*}{1,71} \\
\hline Não & 255 & 86,1 & 690 & 89,0 & \\
\hline \multicolumn{6}{|c|}{ DIU cobre ou hormonal } \\
\hline Sim & 20 & 6,8 & 6 & 0,8 & \multirow{2}{*}{32,36} \\
\hline Não & 276 & 93,2 & 769 & 99,2 & \\
\hline \multicolumn{6}{|c|}{ Esterilização cirúrgica } \\
\hline Sim & 28 & 9,5 & 7 & 0,9 & \multirow{2}{*}{49,60} \\
\hline Não & 268 & 90,5 & 768 & 99,1 & \\
\hline \multicolumn{6}{|c|}{ Contracepção de emergência } \\
\hline Sim & 18 & 6,1 & 50 & 6,5 & \multirow[b]{2}{*}{0,049} \\
\hline Não & 278 & 93,9 & 725 & 93,5 & \\
\hline \multicolumn{6}{|c|}{ Outros métodos hormonais } \\
\hline Sim & 36 & 12,2 & 35 & 4,5 & \multirow{2}{*}{20,23} \\
\hline Não & 260 & 87,8 & 740 & 95,5 & \\
\hline Total & 296 & 100 & 775 & 100 & - \\
\hline
\end{tabular}

Fonte: Berteloni GMA, et al., 2014.

Sobre os motivos para evitarem a gestação no momento da pesquisa, esta pergunta ofertava opções de respostas, como: deseja concluir os estudos; não deseja ser mãe; deseja engravidar posteriormente, entre outras, descritas na Tabela 5 e também a possibilidade de descrição de outros motivos não previamente elencados, na forma de questão aberta.

Foi predominante a resposta entre as $\mathrm{MCHO}$ em 34,1\% que já possuíam número de filhos desejados, seguido da resposta em não possuírem condições financeiras em 16,2\%. Entre as nuligestas, 39,1\% desejavam engravidar posteriormente, $25 \%$ desejavam concluir seus estudos e $20,6 \%$ não desejavam serem mães (Tabela 5). 
Tabela 5 - Caracterização dos motivos que as levam a evitar a gestação, n=1071. Brasil, 2014.

\begin{tabular}{|c|c|c|c|c|c|}
\hline Variáveis & $\begin{array}{c}\text { MCHO } \\
\text { N }\end{array}$ & $\%$ & Nuligestas & $\%$ & Valor de $p$ \\
\hline Deseja concluir os estudos & 43 & 14,5 & 196 & 25,3 & \\
\hline Não deseja ser mãe & 12 & 4,1 & 160 & 20,6 & \\
\hline Deseja engravidar posteriormente & 38 & 12,8 & 303 & 39,1 & \\
\hline Possui quantidade de filhos desejada & 101 & 34,1 & - & - & \\
\hline Não possui condições financeiras & 48 & 16,2 & 47 & 6,1 & 441,40 \\
\hline Não possui companheiro fixo & 10 & 3,4 & 45 & 5,8 & \\
\hline Esterilização cirúrgica & 21 & 7,1 & 5 & 0,6 & \\
\hline Motivos de saúde & 11 & 3,7 & 6 & 0,8 & \\
\hline Outros & 12 & 4,1 & 13 & 1,7 & \\
\hline
\end{tabular}

Fonte: Berteloni GMA, et al., 2014.

\section{DISCUSSÃO}

O perfil das mulheres, em sua integralidade, está além das suas questões biológicas e estritamente reprodutivas. Com as mudanças comportamentais, a representação feminina e os motivos para evitar a gestação moldam a mulher que está em adaptação frente às novas necessidades, escolhas e opções de vida, concomitante na era da modernização digital, e com o acesso rápido da informação pela internet (BRAGA RC, et al., 2018).

Esse estudo pesquisou o perfil da saúde das mulheres usuárias da internet e os motivos que às levam a evitar a gestação. Do modo geral, não houve diferença significativa em relação aos motivos de evitarem uma gestação entre os grupos das mulheres com histórico obstétrico (MCHO) e entre as nuligestas.

Sobre ao perfil das mulheres pesquisadas houve predominância de um perfil jovem, entre 14 e 29 anos em $87 \%$ das nuligestas e $65 \%$ delas com ensino superior, uma pequena diferença entre o grupo das MCHO, sendo 41,9\% com ensino superior. Em comparação com o Relatório Anual Socioeconômico da Mulher RASEAM os dados divulgados são semelhantes, pois não classificaram por idade e apresentaram a média de $54,6 \%$ das mulheres com ensino superior (BRASIL, 2018).

Possuir companheiro foi relatado no presente estudo em $83,4 \%$ das $\mathrm{MCHO}$ e em $58,6 \%$ das nuligestas. A análise da distribuição do percentual das famílias brasileiras é menor do que o encontrado nesse estudo, com $40,9 \%$ das mulheres sem cônjuge e $23,2 \%$ casais com filhos e as mulheres nuligestas com companheiro apresentaram apenas 10,6\% (BRASIL, 2018).

Em relação situação ocupacional, $79,4 \%$ das $\mathrm{MCHO}$ e $52,5 \%$ das nuligestas, exerciam atividades remuneradas, representando mais da metade das mulheres pesquisadas, acima da média brasileira. Sem distinção pelo histórico obstétrico, pesquisas populacionais no Brasil apresentam dados de $65,1 \%$ das mulheres não possuirem atividade remunerada. Em comparação a distribuição de emprego formal e grau de escolaridade, demonstra a relação do ensino superior contribuir para o emprego formal em $58,9 \%$ das mulheres (SÃO PAULO, 2017).

Quanto ao acesso aos serviços de saúde nos dois grupos, $\mathrm{MCHO}$ e nuligestas, apresentaram predominância na utilização dos serviços públicos de saúde em mais de $60 \%$, isto reflete os esforços, a abrangência e a crescente amplitude das ações em saúde pelas políticas públicas voltadas às mulheres (BRASIL, 2013).

O consumo de tabaco foi minoria respectivamente em $8,8 \%$ das $\mathrm{MCHO}$ e $6,6 \%$ das nuligestas do qual a proporção foi equivalente à média das mulheres brasileiras. Numa visão integral sobre a saúde das mulheres, o tabagismo é um dos principais fatores de risco evitáveis para diminuição da mortalidade. Em 2014, a média brasileira correspondia a $11,2 \%$ e na pesquisa realizada em 2017 , no Brasil, contatou-se que $7,5 \%$ das mulheres eram tabagistas, sendo significativa a redução do consumo para sua saúde (GONÇALVES T, et al., 2019; IBGE, 2013; BRASIL, 2018). 
As proporções de sobrepeso e obesidade corresponderam a quase $50 \%$ das $\mathrm{MCHO}$ e em mais de $35 \%$ nas nuligestas, o sedentarismo sobressaiu-se na maioria das mulheres, mais da metade estavam com o peso adequado, sendo eutróficas em relação a mensuração sobre o Índice de Massa Corpórea. No entanto, não corresponde apenas à massa magra, e diante da ausência de exercício, o cuidado de saúde está longe do ideal de um estilo de vida saudável.

A prática de exercício físico acima de três vezes na semana, considerando um tempo mínimo de 150 minutos por semana, pode oferecer maiores benefícios para a saúde, de forma integral também para população feminina, e colaborar com a qualidade e expectativa de vida, e principalmente colaborar para a prevenção de gestação de alto risco (BRASIL, 2017; GUIMARÃES AKRC, 2018).

$\mathrm{Na}$ análise da saúde reprodutiva das mulheres neste estudo, mais de $85 \%$ das mulheres afirmaram uso de algum método anticoncepcional para evitar gestação não planejada, 93,0\% das MCHO e 86,6\% das nuligestas. Em relação aos resultados obtidos sobre o uso de contraceptivos, ressalta-se que faz parte da recomendação dos profissionais, a dupla proteção, sendo para a prevenção da gestação, e grande parte das Infecções Sexualmente Transmissíveis (IST) (FERRERA APC, et al., 2019).

No entanto, o uso de preservativo como método principal de barreira foi presente em $51,9 \%$ das nuligestas, e $65,5 \%$ das $\mathrm{MCHO}$ não o utilizam. Isto ainda demonstra a vulnerabilidade das mulheres na conduta passiva e negligente com a sua saúde frente ao parceiro íntimo em proteger-se apenas de uma gestação com métodos anticoncepcionais, e não serem protagonistas para sua prevenção às IST (BRASIL, 2013).

$\mathrm{O}$ uso de métodos de barreira pelas mulheres, neste estudo, ainda foi maior do que encontrado na literatura, que demonstrou uma proporção de uso de métodos de barreira em 16,2\% numa população com uso predominante do sistema de saúde privado, e principalmente em mulheres solteiras (FERRERA APC, et al., 2019).

O não uso de preservativo constitui um problema de saúde pública, no que diz respeito às IST. Esperavase que o uso fosse maior nesta população, comparada a outros estudos, devido ao fato destas mulheres serem potencialmente mais ativas no acesso à informação com a internet, porém, considerando os tipos de relacionamentos, enquanto estáveis e esporádicos, os casais tendem a descartar o uso do preservativo ao longo do relacionamento (PEREIRA NA, et al., 2016; GUIMARÃES AKRC, 2018).

Neste sentido, as mulheres podem utilizar anticoncepcional hormonal oral, com o qual seria indicado associar o preservativo, ou na presença de esquecimento das drágeas, seria recomendado do mesmo modo o uso do preservativo associado. Em outra situação, pós-coito indesejado ou inseguro, poderia utilizar o contraceptivo de emergência. Mesmo com estas orientações, ainda há mulheres que recorrem ao coito interrompido, artifício de baixa eficácia e que não protege contra as IST, para minimizar a chance da gestação não planejada (BASTOS LL, et al., 2014).

Em relação aos outros métodos contraceptivos utilizados, o uso de anticoncepcional hormonal oral foi descrito em $43,6 \%$ das $\mathrm{MCOH}$ e em $62,5 \%$ das nuligestas. O ranking de métodos utilizados pelas mulheres brasileiras difere das outras literaturas somente quanto ao uso do preservativo, que geralmente é menor, os demais são utilizados em menor proporção, como neste estudo (SCAVUZZI A, et al., 2016).

Entretanto, o método anticoncepcional hormonal oral e o preservativo são métodos que as mulheres precisam ter disciplina, pois o esquecimento eventual de uma drágea ou a não utilização do preservativo em todas as relações sexuais, pode ser o suficiente para ocasionar uma gestação não planejada ou não desejada. É neste momento, que algumas mulheres também recorrem ao coito interrompido e/ou à contracepção de emergência, ou até mesmo ao abortamento, quase sempre clandestino e inseguro (BRANDÃO ER e CABRAL CS, 2017).

Vale considerar que a contracepção de emergência não é exatamente um método, e sim, uma alternativa à falha da anticoncepção, sendo um importante recurso contra as gestações não planejadas e não desejadas, e consecutivamente, ao abortamento provocado. Já o coito interrompido, tem-se desaconselhado sua prática devido à baixa eficácia quanto à prevenção da gestação e riscos de contrair uma IST (OLSEN JM, et al., 2018; FERRERA APC, et al., 2019). 
Em outros estudos brasileiros o índice de esterilização cirúrgica é relativamente maior em comparação aos outros métodos. Destaca-se que neste estudo houve a participação de $\mathrm{MCHO}$ jovens para as quais não seria indicado este método como primeira escolha (BRANDÃO ER, 2019). Sobre os motivos elencados pelas mulheres para evitar a gestação nas $\mathrm{MCHO}$, o principal motivo foi por terem atingido a quantidade de filhos que desejava em $34,1 \%$, seguido por não terem condições financeiras em $16,2 \%$ e do mesmo modo, com $14,5 \%$ terem planos de concluir os estudos.

Contudo, nas nuligestas, os motivos principais para evitar a gestação estavam interligados em $39,1 \%$ desejo de terem filhos posteriormente, e em $25,3 \%$ das nuligestas desejavam a conclusão acadêmica antes de terem filhos. Percebe-se, em destaque, que os objetivos atuais das mulheres que desejam ter filhos, vêm após as realizações pessoais, relacionadas à carreira acadêmica e profissional (BRAGA RC, et al., 2018). Na análise das $20,6 \%$ das nuligestas que não desejavam terem filhos nesse estudo, pode levar a reflexão sobre os novos hábitos e a constituição familiar singular atual, como um novo olhar sobre o papel das mulheres na sociedade, e uma confirmação da nova identidade feminina (MARCOS CM, 2017).

Em um estudo realizado sobre a subjetividade da mulher, realizado por psicólogas, concluíram que não necessariamente ser mulher é ser mãe. Sendo que o desempenho profissional foi apontado como importante na realização pessoal. Além de enfatizar a relatividade da maternidade como objetivo secundário de vida, e priorizarem a independência e individualidade não interferindo na feminilidade e da sexualidade das mulheres (BRAGA RC, et. al., 2018).

Por outro lado, a postergação da gestação deve ser considerada não apenas pelo lado positivo do protagonismo e empoderamento da mulher, deve ser de conhecimento das mulheres que ao postergar a gestação pode emergir outras necessidades e riscos potenciais à saúde. Evidências de estudos apontam que mulheres que engravidam em idade avançada, superior a 35 anos, apresentam maior probabilidade de desfechos com complicações maternas e fetais em relação a mulheres mais jovens e, ainda, as que optam por nunca engravidarem podem ter um maior risco de alguns tipos de câncer, como o de mamas (BRASIL, 2013; OLIVEIRA, 2020).

Gestar é um papel determinado à mulher como natural e biologicamente condicionado, a sexualidade não deve ser aplicada apenas com o foco para aumento de prole. Esta imposição da maternidade como um papel social feminino padronizado e esperado como comportamento aceitável não deve ser para caracterizar todas as mulheres. Mediante as mudanças de comportamento da mulher, a sociedade deve respeitar as mulheres que possuem uma identidade feminina com protagonismo, do qual buscam dos seus desejos individuais além da maternidade (BRAGA RC, et. al., 2018). As mulheres devem realizar suas escolhas baseadas em seus ideais de vida, podem postergar a maternidade para o momento considerado oportuno por elas para gestar mediante análise de riscos de saúde, ou também aceitar o desejo que não visualizam sua vida condicionada a ser mãe (MACHADO JSA, et al, 2020).

\section{CONCLUSÃO}

O perfil da saúde das mulheres na internet colabora para possibilidades de empoderamento e de acesso à informação. Não houve resultados significativos entre as mulheres com histórico obstétricos e nuligestas ao correlacionar seu protagonismo com demais pesquisas brasileiras. Os métodos anticoncepcionais mais utilizados foram o hormonal oral e o preservativo. Os perfis das mulheres continuam em adaptação aos novos modelos de vida, demonstram motivos relevantes para evitar a gestação, relacionados aos objetivos individuais e possuem um uso difundido dos métodos anticoncepcionais reversíveis para postergação da maternidade. Os serviços de saúde devem garantir acesso aos métodos e informações adequadas sobre a contracepção, para prevenção de agravos à saúde da mulher, advindos de gestações não planejadas.

\section{REFERÊNCIAS}

1. AZEVEDO AF. Direito ao aborto, gênero e a pesquisa jurídica em direitos fundamentais. Sexualidade Saúde e Sociedade, 2017; 26:236-261.

2. BASTOS LL, et al. O acesso à contracepção de emergência como um direito? Os argumentos do consórcio internacional sobre contracepção de emergência. Interface - Comunicação, Saúde, Educação, 2014;18(48):37-46. 
3. BRAGA RC, et al. Para Além Da Maternidade: As Configurações do desejo na mulher contemporânea. Revista da Graduação em Psicologia da PUC Minas, 2018;3(6):523-40.

4. BRANDÃO ER e CABRAL CS. Da gravidez imprevista à contracepção: aportes para um debate. Caderno de Saúde Pública, 2017; 33 (2).

5. BRANDÃO, ER. Métodos contraceptivos reversíveis de longa duração no Sistema Único de Saúde: o debate sobre a (in)disciplina da mulher. Ciência \& Saúde Coletiva, 2019;24(3), 875-879.

6. BRASIL. Constituição da República Federativa do Brasil. Constituição. Brasília, DF, 1988.

7. BRASIL. lei № 9.263, de 12 de janeiro de 1996. Regula o $§ 7^{\circ}$ do art. 226 da Constituição Federal, que trata do planejamento familiar, estabelece penalidades e dá outras providências. Brasília, DF, 1996.

8. BRASIL. Secretaria de Atenção à Saúde. Saúde sexual e saúde reprodutiva. Brasília: Ministério da Saúde, 2013; 300 p.

9. BRASIL. Secretaria de Vigilância em Saúde. Vigitel Brasil 2017: vigilância de fatores de risco e proteção para doenças crônicas por inquérito telefônico : estimativas sobre frequência e distribuição sociodemográfica de fatores de risco e proteção para doenças crônicas nas capitais dos 26 estados brasileiros e no Distrito Federal em 2017. Brasília: Manistério da Saude; 2018, 130 p.

10. BRASIL. Secretaria Nacional de Políticas para Mulheres. Relatório Anual Socioeconômico da Mulher. 1aㅡ impressão. Brasília: Secretaria Nacional de Políticas para Mulheres, 2018.

11. COSTA FA. Mulher, trabalho e família: os impactos do trabalho na subjetividade da mulher e em suas relações familiares. Revista da Graduação em Psicologia da PUC Minas, 2018; 3 (6) 434-452.

12. CUNHA MS, VASCONCELOS MR. Fecundidade e participação no mercado de trabalho brasileiro. Nova Economia, 2016; 26(1):179-206.

13. DINIZ D, et al. National Abortion Survey 2016. Ciência Saúde Coletiva, 2017;22(2):653-60.

14. FERRERA APC, et al. O. (Des)conhecimento de mulheres sobre a utilização de métodos contraceptivos. Revista de enfermgem UFPE on line, 2019;13(5):1354-60.

15. GONÇALVES T, et al. Social inequalities in the use of contraceptives in adult women from Southern Brazil. Revista de Saúde Pública, 2019; 53,28.

16. GUIMARÃES AKRC, et al. Obesidade na gestante adolescente: uma revisão bibliográfica. Revista Eletrônica Acervo Saúde, 2018;(18), e131.

17. IBGE. Pesquisa Nacional de Saúde 2013. Brasília: Ministério da Saúde, 2014; 181 p.

18. JUSTINO GBS, et al. Saúde sexual e reprodutiva no puerpério: vivências de mulheres. Revista de Enfermagem UFPE on line, 2019;13:e240054.

19. MACHADO JSA, et al. Cinderela de sapatinho quebrado: maternidade, não maternidade e maternagem nas histórias contadas pelas mulheres. Saúde em Debate, 2020; v. 43, n. 123: 1120-1131.

20. MACHADO JSA, PENNA CMM. Reprodução feminina e saúde sob os olhares de mulheres sem filhos. Rev Min Enferm. 2016; 20: e972.

21. MARCOS, CM. O desejo de ter um filho e a mulher hoje. Trivium - Estudos Interdisciplinares, 2017; 9(2), 246-256.

22. MORETTI FA, et al. Access to health information on the internet: a public health issue? Revista da Associação Médica Brasileira, 58(6): 650-658.

23. OLIVEIRA DN, et al.Desfechos obstétricos em gestações tardias no estado de Minas Gerais. Revista Eletrônica Acervo Enfermagem, 2020; (3) e2555.

24. OLIVEIRA ECS, et al. Ás margens do cuidado: regulações de gênero em uma equipe de saúde, Physis: Revista de Saúde Coletiva, 2018;28(2): e280208.

25. OLSEN JM, et al. Young women's contraceptive practices: a household survey in the city of São Paulo, Brazil. Cadernos de Saúde Publica, 2018;34(2): e00019617.

26. PEREIRA NA, et al. Internet, geração Y e saúde: um estudo nas comunidades de Manguinhos (RJ). Comunicação \& Informação, 2016;19(1), 20-36.

27. RODRIGUES LSA, et al. Planned parenthood: heterosexual women's perceptions about the role of the couple. Revista de enfermagem UFPE on line, 2014; 8(2):323-9.

28. SÃO PAULO, Centro de Estudos Sindicais e Economia do Trabalho. Caderno de formação. Mulheres: mundo do trabalho e autônomia. 2017, Caderno 3: 1-50.

29. SCAVUZZI A, et al. Continued Compliance and Degree of Satisfaction in Nulligravida and Parous Women with Intrauterine Contraceptive Devices. Revista Brasileira de Ginecologia e Obstrícia, 2016;38(03):132-9.

30. SEABRA ACCM e DUSSE F. Feminismo e redes sociais: um estudo sobre empoderamento pela internet. Interfacis, 2017;3 (1)

31. SILVA MMJ, et al. Anxiety in pregnancy: prevalence and associated factors. Revista da Escola de Enfermagem da USP, 2017; $51: e 03253$. 